\title{
Spontaneous Rupture of the Extensor Pollicis Longus Tendon
}

\author{
Chul Han Kim
}

Department of Plastic and Reconstructive Surgery, Soonchunhyang University College of Medicine, Seoul, Korea

Correspondence: Chul Han Kim

Department of Plastic and Reconstructive Surgery, Soonchunhyang University College of Medicine, 59 Daesagwan-ro, Yongsan-gu, Seoul 140-743, Korea Tel: +82-2-709-9283, Fax: +82-2-796-3543

E-mail:kchann@hanmail.net

This work was supported in part by the Soonchunhyang University Research Fund.

No potential conflict of interest relevant to this article was reported.

Received: 2 May 2012 • Revised: 15 Jun 2012 • Accepted: 16 Jun 2012 pISSN: 2234-6163 • elSSN: 2234-6171

http://dx.doi.org/10.5999/aps.2012.39.6.680 • Arch Plast Surg 2012;39:680-682

Copyright (C) 2012 The Korean Society of Plastic and Reconstructive Surgeons This is an Open Access article distributed under the terms of the Creative Commons Attribution Non-Commercial License (http://creativecommons.org/licenses/by-nc/3.0/) which permits unrestricted non-commercial use, distribution, and reproduction in any medium, provided the original work is properly cited.

Spontaneous rupture of the extensor pollicis longus (EPL) tendon at the wrist is uncommon and mainly reported after fracture of the distal radius at Lister's tubercle, in synovitis, tenosynovitis, or rheumatoid arthritis [1]. Other causes include steroid treatment, excessive abnormal motion of the wrist joint, bony spur developing after distal radius or scaphoid fracture, misplaced external fixator, nonunion of the scaphoid, and subluxation of the distal ulna [2,3]. However, it has rarely been reported in association with occupational work activity without predisposing factors [4]. We report a case of spontaneous rupture of the EPL in a cook without rheumatoid arthritis, tenosynovitis, or steroid treatment.

A 41-year-old female visited to our clinic with a loss of full extension of the interphalangeal joint in the left thumb. She had been intensively engaged as a

Fig. 1.

Preoperative findings. A 41-year-old woman presented with an inability to extend the interphalangeal joint of the left thumb.

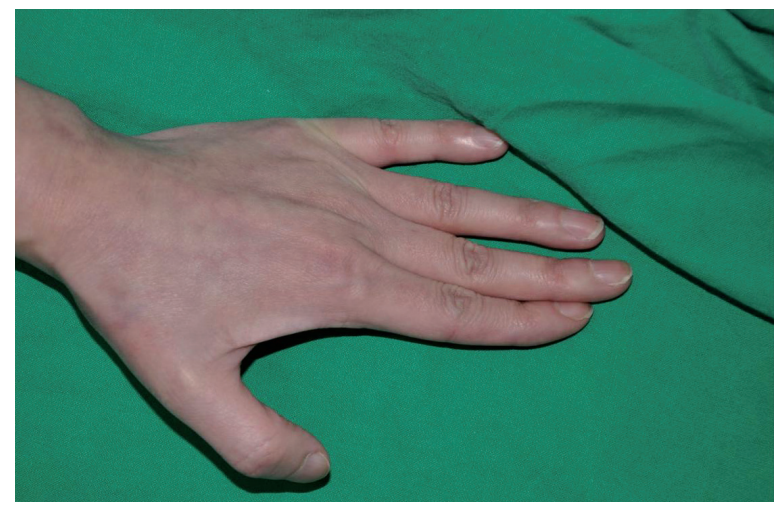




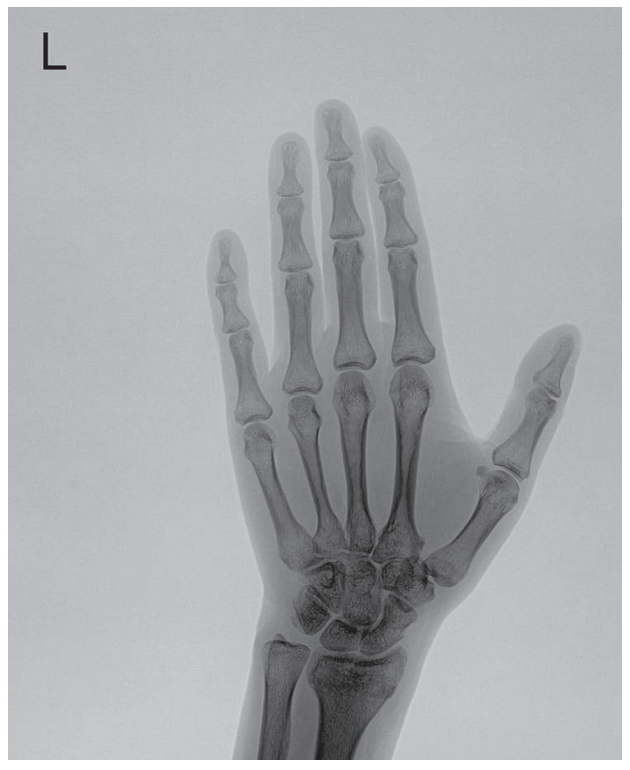

Fig. 2.

An X-ray of the wrist demonstrated no significant findings such as rheumatoid arthritis, distal radius, or carpal fracture.

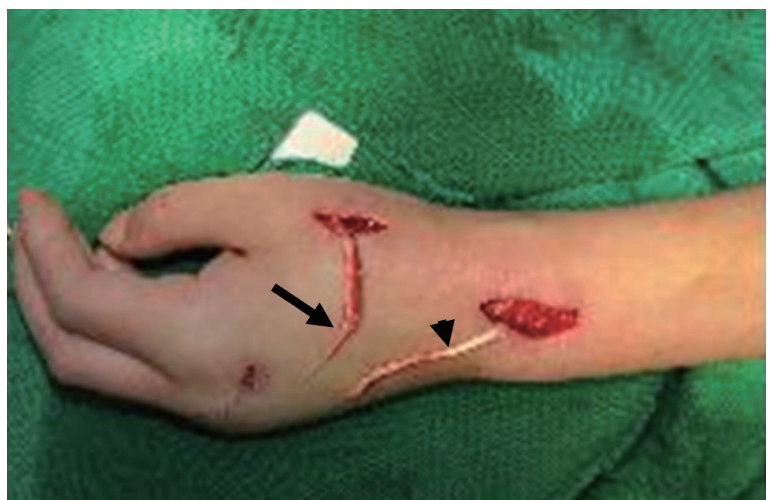

Fig. 3.

An intraoperative view revealed the frayed distal portion of the spontaneously ruptured extensor pollicis longus tendon (black arrow) at the level of the dorsal radial tubercle. The extensor indicis proprius tendon (black arrowhead) was harvested. restaurant cook for the past 8 years. She felt pain in the left wrist when cooking 3 weeks earlier. Before this accident, she sometimes experienced mild pain at the wrist, but she had no difficulty in her daily life. She had no history of recent severe trauma, rheumatoid arthritis, or steroid medication. On physical examination, flexion of the interphalangeal joint of the thumb was possible but loss of active full extension was identified (Fig. 1). X-ray demonstrated no significant findings such as rheumatoid arthritis, or distal radius or carpal fracture (Fig. 2). The routine laboratory results were in the normal range. She tested negative for the rheumatoid factor and had no symptoms of rheumatoid arthritis or gout. On sonography, there was no sign of tenosynovitis showing tendon thickening or hypoechogenicity with anechoic fluid collection, and this confirmed rupture of the EPL tendon.

During the surgery, the distal portion of the ruptured EPL tendon was found at Lister's tubercle. It was grossly frayed and adhered to the surrounding tissue, but there was no macroscopic tenosynovitis or synovitis. The proximal end was not identified due to retraction into the forearm. To reconstruct the EPL tendon, the extensor indicis proprius (EIP) tendon was transferred at the dorsum of the second metacarpophalangeal (MCP) joint, and the remaining distal end of the EIP was sutured to the extensor digitorum communis tendon (Fig. 3). The proximal end of the EIP tendon was fixed to the distal stump of the EPL tendon using Pulvertaft's technique at the proximal MCP joint. The tension was set by suturing the EIP tendon to the distal portion of the EPL tendon with the thumb held in $0^{\circ}$ of extension at the interphalangeal and MCP joints and with the wrist in neutral position. The tension was considered satisfactory when full thumb extension occurred with retraction of the reconstructed tendon proximally and with the tenodesis effect of wrist flexion, and when full flexion of the thumb was possible with wrist extension. She was immobilized in a short-arm splint with the wrist in neutral position and the thumb in abduction and extension for 4 weeks, and then gentle range of motion exercise was begun with intermittent splint protection. After 6 weeks postoperatively, the splint was completely removed. At 3 months postoperatively, she showed the full ability to extend the interphalangeal joint of the thumb (Fig. 4).

Rheumatoid arthritis, steroid injection, tenosynovitis, synovitis, and distal radial fracture are well known to be common causes of the spontaneous rupture of the EPL tendon $[1,2]$.

However, spontaneous EPL tendon rupture caused by repeated movement of the wrist joint in association with occupational work activity, without a history of severe trauma, rheumatoid arthritis, or tenosynovitis has rarely been reported [4]. It is particularly unusual that the occupation of cooking 
Fig. 4.

Postoperative view. (A) Flexion was slightly limited. (B) The extension of the interphalangeal joint of the thumb was fully recovered.
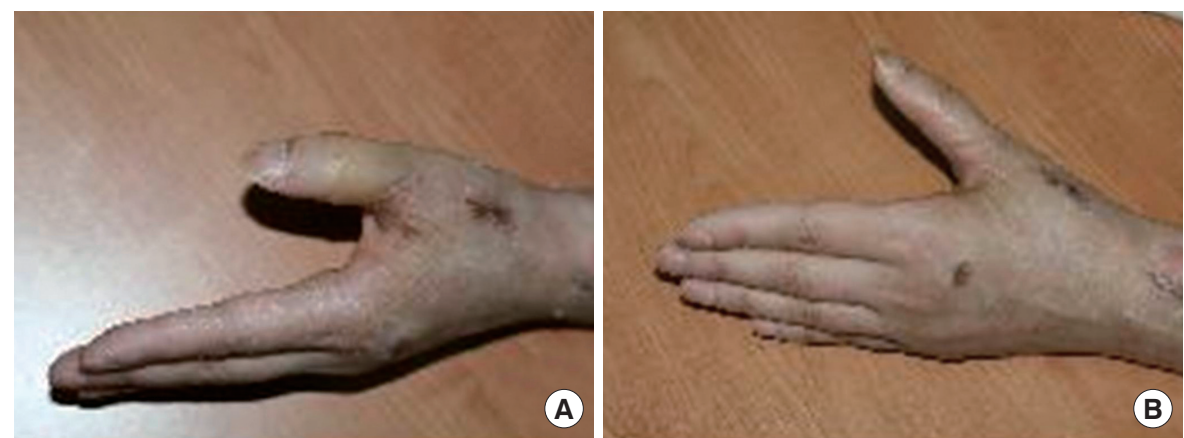

precipitated the spon- taneous rupture of the EPL tendon without other predisposing risk factors. Zvijac et al. [5] reported that the EPL tendon could spontaneously rupture due to attrition of the tendon around Lister's tubercle.

Two major theories of mechanical and vascular factors have been suggested to explain the pathogenesis of spontaneous rupture of the EPL tendon [1, 2 . According to the mechanical theory, the anatomical configuration of the EPL makes it vulnerable to disruption because it courses over the dorsal cortex around Lister's tubercle. The rough bony edge at the distal radius fracture line abrades the EPL tendon, which acts as a potential mechanical factor [2]. The vascular theory says that the increased pressure within the tendon sheath at Lister's tubercle causes ischemia and delayed rupture of the EPL tendon [1]. Microangiographic study revealed that the EPL had a proximal and a distal blood supply with a relatively avascular zone around Lister's tubercle [1]. This zone is supplied its nourishment mainly via diffusion from synovial fluid into the tendon sheath [2]. This synovial nutrition can be compromised in circumstances of increased pressure on the surrounding tendon sheath, such as due to edema or hematoma following fracture or due to inflammation in rheumatoid arthritis $[1,2]$. A decrease in vascularization results in degeneration of the tendon and a minor stress is prone to rupture later. Our patient had been intensively working as a cook for 8 years. It was concluded that prolonged mechanical attrition by repeated use of the wrist damaged the EPL tendon around Lister's tubercle, and the long-term stress of the weakened tendon caused the EPL rupture because she had no trauma, rheumatoid arthritis, or tenosynovitis.
In our patient, direct repair or tendon graft was not possible because the ruptured EPL tendon had retracted into the forearm. Tendon transfer using the EIP was used because it has an appropriate direction and greater excursion. The present case produced a satisfactory result with a tendon transfer of the EIP, and the patient was able to return to working as a cook even though there was a slight limitation of flexion.

This case described the spontaneous rupture of the EPL tendon. Spontaneous rupture of the EPL tendon is rather rare when it occurs in occupational work activity without injury to the underlying bone or arthritic changes. It is postulated that the EPL tendon was weakened by prolonged repetitive activity causing long-term mechanical attrition at work and subsequently ruptured.

\section{References}

1. Engkvist O, Lundborg G. Rupture of the extensor pollicis longus tendon after fracture of the lower end of the radius: a clinical and microangiographic study. Hand 1979;11:76-86.

2. Bjorkman A, Jorgsholm P. Rupture of the extensor pollicis longus tendon: a study of aetiological factors. Scand J Plast Reconstr Surg Hand Surg 2004;38:32-5.

3. Harvey FJ, Harvey PM. Three rare causes of extensor tendon rupture. J Hand Surg Am 1989;14:957-62.

4. Choi JC, Kim WS, Na HY, et al. Spontaneous rupture of the extensor pollicis longus tendon in a tailor. Clin Orthop Surg 2011;3:167-9.

5. Zvijac JE, Janecki CJ, Supple KM. Non-traumatic spontaneous rupture of the extensor pollicis longus tendon. Orthopedics 1993;16:1347-50. 